\title{
Dimensionality of trust: An analysis of the relations between propensity, trustworthiness and trust
}

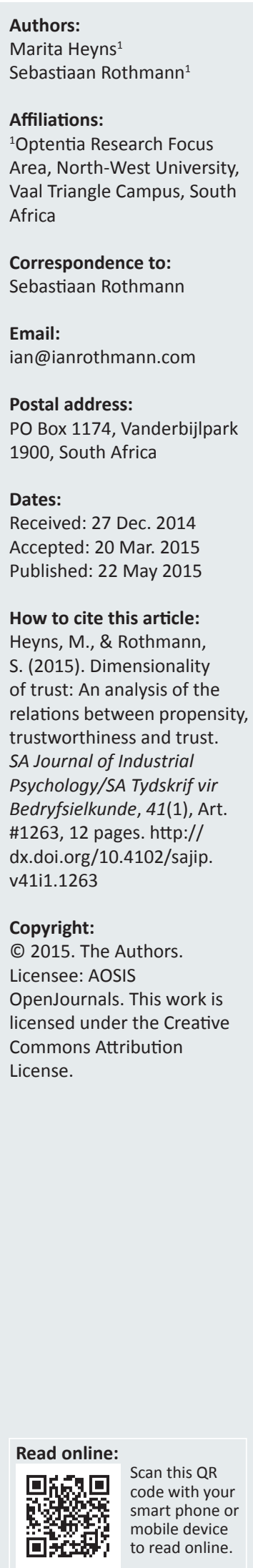

Authors:

Marita Heyns

Rothmann

Area, North-West University,

Vaal Triangle Campus, South

Correspondence to:

Rothman

Email:

Postal address:

PO Box 1174, Vanderbijlpark

Dates:

Received: 27 Dec. 2014

Accepted: 20 Mar. 2015

How to cite this article:

Heyns, M., \& Rothmann

S. (2015). Dimensionality

rust: An analysis of the

trustworthiness and trust.

SA Journal of Industrial

\#1263, 12 pages. http://

dx doi.org/10.4102/sajip.

Copyright:

Commons Attribution

License.
Orientation: Research concerning trust relationships on the interpersonal level, particularly when studied in dyadic relationships from the follower's point of view, is relatively scarce. Only a few researchers have attempted to link multiple dimensions of trust in the same study.

Research purpose: This study examined the dynamic interplay between trust propensity, trustworthiness beliefs and the decision to trust, as perceived within dyadic workplace relationships.

Motivation for the study: No studies, as far as the authors are aware, have ever attempted to use a combination of Mayer and Davis's well-known assessment of trustworthiness and Gillespie's measure of behavioural trust within the same study. By including measures of main antecedents and the actual decision to trust in the same study, the multidimensionality of trust can be established more concretely.

Research approach, design and method: A cross-sectional survey design with a convenience sample $(N=539)$ was used. The Behavioural Trust Inventory and the Organisational Trust Instrument were administered.

Main findings: Results confirmed the distinctness of propensity, trustworthiness and trust as separate main constructs. Trust was strongly associated with trustworthiness beliefs. Trustworthiness beliefs fully mediated the relationship between propensity and trust. The observed relations between propensity and trustworthiness suggest that individuals with a natural predisposition to trust others will be more inclined to perceive a specific trust referent as trustworthy.

Practical/managerial implications: Leaders should realise that their attitudes and behaviour have a decisive impact on trust formation processes: if they are being perceived as trustworthy, followers will be likely to respond by engaging in trusting behaviours towards them. Tools to assess followers' perceptions of the trustworthiness of the leader may provide useful feedback that can guide leaders.

Contribution/value-add: This study contributes to scientific knowledge regarding the influence of propensity to trust and trustworthiness on trust of leaders.

\section{Introduction}

The ability to establish, nurture and restore trust is vital to leadership success in the new global economy (Covey, 2006; Green 2012; Salamon \& Robinson, 2008). The modern workplace has undergone some dramatic changes that, in effect, have reduced reliance on traditional bases of power such as derived from formal positions of authority. Drivers of change such as globalisation, diversity and technological innovations brought about an increased emphasis on the interaction and self-directedness of employees, as well as more flexible work structures that are difficult to exercise control over (Green, 2012; Grey \& Garsten, 2001). Changes affect macro, meso and micro systemic levels of organisations and tend to create an increased reliance on complex matrix systems of authority to resolve multiple and often competing demands for limited resources (Greyvenstein \& Cilliers, 2010). Leadership not only demands the ability to empower both leaders and followers to function effectively within a matrix system, but also implies awareness of multiple leadership roles, the ability to move between different types and styles of leadership and advanced skills to manage complex interpersonal relationships (Greyvenstein \& Cilliers, 2010). Ultimately, changes in the workplace environment have led to an increased reliance on trust as a mechanism to coordinate and control interdependent activities, for in such a dynamic and fast-changing environment it is impossible to contract everything (Gambetta, 2008; Sydow, 2008; Tichy \& Bennis, 2007).

An overwhelming body of research has established that trust indeed contributes significantly to leader effectiveness (Bachraim \& Hime 2007; Douglas \& Zivnuska, 2008; Wasti, Tan, Brower 
\& Önder, 2007). Employee decisions to trust a direct leader are furthermore highly influenced by the character, words and actions of the leader (Costigan et al., 2007; Dirks \& Ferrin, 2002; Tan \& Lim, 2009). In fact, research has shown that the seniority of the position of a trusted party influences the relative importance attached to characteristics: the more senior the position, the more influential the consideration of characteristics becomes in the decision to trust (Burke, Simms, Lazzara \& Salas, 2007). It is therefore important that leaders should put in a concerted effort to build and improve trust relationships with their followers (Burke et al., 2007).

The ability to inspire trust is, however, not an easy task to accomplish. Infact, theincapability toenthuse trust is often listed as one of the most important reasons why leaders fail (Burke, 2006; Pienaar, 2009). The 2011-2012 Kenexa High Performance Institute WorkTrends report (Kenexa, 2012) established that only $48 \%$ of all employees who participated in this worldwide survey trusted their leaders, whilst $28 \%$ actively distrusted their leaders and $24 \%$ were undecided. South African-based research showed varied findings. Previous research on general followership's experiences of organisational leadership revealed that followers have a negative leadership view and also highlighted other themes including an idealisation of the past and blaming of the present, obsession with race and gender and a constantly changing identity (Greyvenstein \& Cilliers, 2010). Regarding an analysis of trust relationships specifically, Bachraim and Hime (2007) found that workplace trust is moderately high, with the highest levels of trust being in supervisors. In contrast, research by Esterhuizen and Martins (2008) points to the existence of a significant trust gap between employees in general and their employers. In yet another study, Steinman and Martins (2009) attempted to identify the 10 key problem areas that impede team functioning in South African organisations, and found a lack of trust in superiors, higher authority and colleagues to be amongst the most important reasons for poor performance. Furthermore, Van der Ohe and Martins (2010) found significant differences in the levels of organisational trust between government participants and other sectors.

Both the significance of trust for meaningful interpersonal relationships and the difficulty of inspiring trust in others underscore the importance of understanding trust-building as a management intervention. In view of the above findings, it is important to realise that specific leader behaviours are quite influential in creating a history of experiences that serve to either build or erode trust in leadership. Kramer (2006) succinctly reminds us that 'although trust may be desirable in the abstract, it really makes sense only when those trustees on whom people depend are deserving of that trust' (pp. 10-11).

\section{A model of trust}

Different conceptualisations of trust have hindered previous research on trust, particularly due to a lack of clear differentiation amongst factors that contribute to trust, trust itself and the outcomes of trust (Mayer, Davis \& Schoorman, 1995).
Mayer et al. (1995) attempted to clarify some of this confusion. Their multidimensional definition of trust is one of the most widely accepted (Rousseau, Sitkin, Burt \& Camerer, 1998; Wasti et al., 2007) and most cited definitions of trust in the organisational science literature (Ball, 2009; Gillespie, 2012). They define trust as:

the willingness of a party to be vulnerable to the actions of another party based on the expectation that the other will perform a particular action important to the trustor, irrespective of the ability to monitor or control that other party. (Mayer et al., 1995, p. 712)

Werbel and Henriques (2009, p. 781) simplify this to 'the willingness to make oneself vulnerable to another person despite uncertainty regarding motive and prospective actions', thereby underscoring the fact that risk-taking, vulnerability and uncertainty are core elements of a trusting relationship.

Mayer et al. (1995) and Schoorman, Mayer and Davis (2007) developed an extensive and integrated model of interpersonal trust that provided a turning point for trust research (Ball, 2009). By 2009, Mayer et al.'s seminal work had been cited in the literature more than 2900 times (Ball, 2009) and by 2013 more than 149000 times (Google Scholar, 2013). Their model was one of the first to conceptualise trust as a multidimensional concept that is essentially relational or interpersonal and context-specific in nature: rather than seeing trust merely as an individual characteristic that remains constant regardless of context, their model defines trust as relational, therefore largely dependent on characteristics of both the trustor (the person who trusts) and the trustee (the person to be trusted) within a specific relationship that varies in depth and strength over time (Mayer et al., 1995; Schoorman et al., 2007).

Not only does the model account for the interpersonal and possibly reciprocal nature of trust, it also considers the influence of perceived risk and helps to explain why some individuals are trusted more than others (Mayer et al., 1995). The relationships between conditions for trust, trust itself and its outcomes are illustrated in Figure 1 (Mayer et al., 1995).

The core element of the model centres on trust itself, which signifies an intention to accept vulnerability towards another, as is evidenced by the actual decision to trust (Schoorman et al., 2007). The willingness to accept vulnerability towards another party and to take risks within the relationship, is sometimes referred to as 'behavioural trust' (Gillespie, 2003) or 'volitional trust' (Caldwell \& Dixon, 2009; McEvily \& Tortoriello, 2011). Trust can manifest in different forms, such as being willing to rely on or disclose information to a leader (Gillespie, 2003). A proper measure of trust should therefore assess the extent to which the trusting party is willing to allow the trustee to have significant influence over their life (Gillespie, 2003; Schoorman et al., 2007) within a specific context, such as at the place of work (Colquitt \& Rodell, 2011). 


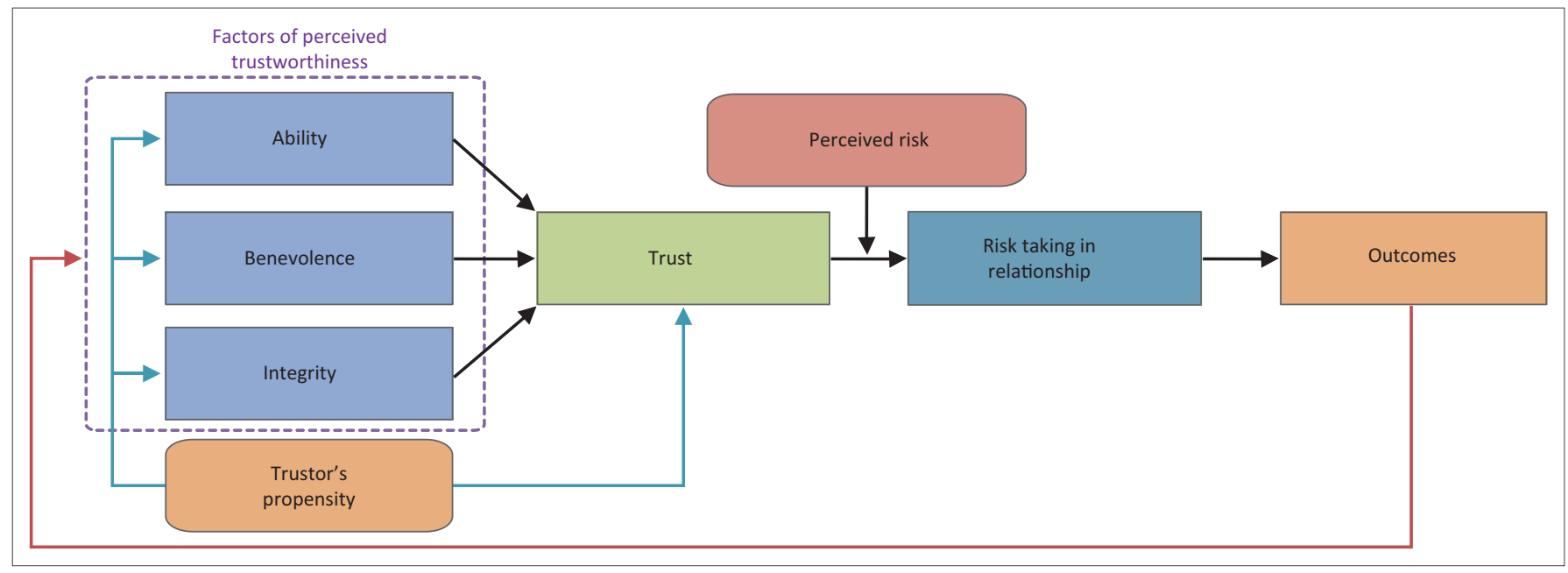

Source: Mayer, R.C., Davis, J.H., \& Schoorman, F.D. (1995). An integrative model of organizational trust. Academy of Management Review, $20,715$.

FIGURE 1: Integrated model of interpersonal trust.

The model furthermore indicates trait factors such as the trustor's propensity and perceptions of trustee characteristics comprising trustworthiness, such as ability, benevolence and integrity, are antecedents of trust (Mayer \& Gavin, 2005). It is therefore important to realise that the antecedents of trust should be differentiated from trust itself (Mayer et al. 1995; Schoorman et al., 2007).

\section{Antecedents of trust}

Propensity indicates a consistent tendency to be willing to trust others across a broad spectrum of situations and trust targets (McKnight, Cumming \& Chervany, 1998). This suggests that every individual has some baseline level of trust that will influence the person's willingness to rely on the words and actions of others. Propensity is thought to drive trusting beliefs, especially when little information about the trustee is known, such as at the early stages of a relationship (Colquitt, Lepine \& Wesson, 2009), and in ambiguous situations (Gill, Boies, Finegan \& McNally, 2005). However, the influence of one's disposition to trust is not limited to such situations, as propensity can still shape trusting beliefs even after more information about the trustee becomes available (Searle et al., 2011). This is because propensity acts like a filter that colours the interpretations of others' actions and perceived trustworthiness, thereby serving as a platform from which the leap of faith to trust can be taken, but retaining the impact of one's predisposition even after trustworthiness can be inferred (Colquitt, Scott \& LePine, 2007).

Trust is also understood as a complex compilation of judgements by the trustor on different characteristics of the trustee. Although numerous characteristics can influence one's judgement of another's trustworthiness, Mayer et al. (1995), after conducting a thorough review of factors that lead to trust, concluded that mainly three characteristics, namely ability, benevolence and integrity, explain a major portion of trustworthiness and are considered to be the most salient (Mayer et al., 1995). Both Burke et al. (2007) and
Colquitt et al. (2009) confirmed through their research that the wider variety of characteristics mentioned in the literature are indeed indicative of the same underlying constructs and essentially relate to perceptions of the trustee's character (expressly integrity), capacity (particularly competence and expertise) and goodwill. According to the 2011-2012 Kenexa High Performance Institute WorkTrends report (Kenexa, 2012), these three antecedents - ability, benevolence and integrity - explain up to $80 \%$ of the decision to trust.

Ability is 'that group of skills, competencies, and characteristics that enable a party to have influence with some specific domain' (Mayer et al., 1995, p. 717); it captures the 'can-do' component of trustworthiness (Colquitt et al., 2007). Benevolence refers to the trustee's intention to act well towards others without having an egocentric profit motive (Schoorman et al., 2007). Benevolence corresponds best to the 'will-do' component of trustworthiness (Colquitt et al., 2007) and is closely associated with synonyms such as loyalty, openness, caring and supportiveness (Mayer et al., 1995). Since 'can-do' does not necessarily result in 'will-do', it is evident that benevolence affects trust separately and independent of the ability component (Colquitt et al., 2007). Benevolent leaders nevertheless maintain perspective in that they strike a balance between a deep personal commitment to the welfare of others whilst simultaneously furthering the best interests of the organisation (Caldwell \& Dixon, 2009). Integrity is defined as 'the trustor's perception that the trustee adheres to a set of principles that the trustor finds acceptable' (Mayer et al., 1995, p. 719). Integrity can be judged by examining previous behaviours, reputation, the similarity between the behaviour of a trustee (e.g. a leader) and the trustor's (e.g. a follower's) internal beliefs, and the consistency between words and actions (Mayer et al., 1995).

Perceptions of trustworthiness influence the degree of risk that a trustor is willing to take within a particular relationship. If a leader is perceived to be untrustworthy, an employee will most likely conclude that trusting such a leader will 
lead to disappointment, as the outcome of trust may have unfavourable consequences for personal psychological safety. The perceived trustworthiness of a trustee is therefore pivotal to enable the necessary cognitive 'leap of faith' for the decision to trust (Möllering, 2006).

\section{Outcomes of trust}

The final component of the proposed trust model (Mayer et al., 1995; Schoorman et al., 2007) addresses the outcomes of trust, which are various types of actual risk-taking in the relationship with the trustee (Mayer \& Davis, 1999). Mayer and Gavin (2005) describe the relation between trust and its outcomes as follows: 'trust is a generalized behavioral intention to take risk, whereas the outcome is actually taking risk' (p. 874). This component also includes a feedback loop, which allows the trustor to evaluate outcomes of previous vulnerability and a subsequent reappraisal of trustworthiness (Mayer \& Gavin, 2005). This feedback loop allows for exogenous factors that can change perceived trustworthiness, such as when the trustor receives information regarding the character of the trustee from a third party. The re-appraisal that may occur in the latter instance is not necessarily directly through increased vulnerability or risk-taking in the relationship, but rather as a result of a learning curve that took place (Mayer \& Davis, 1999).

\section{Relations between components of trust}

Mayer et al. (1995) and Schoorman et al. (2007) emphasise that the antecedents of trust - propensity and trustworthiness beliefs - affect the trust action, but cannot be equated to trust itself. Trustworthiness refers to attributes of a trustee that inspire trust, whilst trusting is something that the trustor does; therefore, trustworthiness and trust are two separate constructs. Gillespie $(2003,2012)$ elaborates on this point by explaining that, even though one may perceive someone else as trustworthy, it does not automatically follow that one would actually engage in trusting behaviour towards that person. In contrast, one's willingness to actively engage in trusting behaviours towards a trust target indicates actual risk-taking within the relationship and should therefore serve as a closer proxy for trust.

A's belief in B's trustworthiness is nevertheless expected to be a strong predictor of A's decision to trust B, since the belief is based on probabilities (Nooteboom, Berger \& Noorderhaven, 1997) and carries a crucial 'strength of feeling' (Bhattacharya, Devinney \& Pillutla, 1998, p. 462) that elevates it above mere hopefulness, blind faith or gullibility (McEvily, Perrone \& Zaheer, 2003). Therefore, even though trustworthiness and trust are two separate concepts, they are very closely related since interpersonal trust cannot exist without a positive assessment of the trustee's trustworthiness.

Colquitt et al. (2007) previously explored the relationship between precursors of trust, trust itself and several organisational outcomes. They conducted an extensive meta-analysis including 132 independent samples, but failed to reach a conclusive answer regarding the strength of the relations between the variables in their study. Obviously, a meta-analysis incorporates the limitations of the studies on which it is built. Another important limitation was the fact that they could only examine the main effects of the variables on trust. The potential effects of propensity on the relation between trustworthiness and trust could not be assessed, because meta-analytical structural equation modelling is ill suited to do this (Colquitt et al., 2007).

The proper exploration of the relation between propensity, trustworthiness and trust implies that a more complete understanding of trust should ideally involve a comprehensive measure that includes an assessment of both the decision to trust as well as its antecedents within the same study. McEvily and Tortoriello (2011) support this view by emphasising the necessity of further research to (1) operationalise trust as a multidimensional construct, (2) empirically assess the extent to which distinct dimensions exist and (3) assess the nature and degree of their relationships with each other. Dietz and Den Hartog (2006) recommend that a comprehensive measure of trust should capture both the respondent's beliefs about the trustee as well as the intention to act on such beliefs. This approach is advisable because, as Dietz and Den Hartog correctly point out, the mere knowledge that someone is considered trustworthy does not necessarily imply that the trustor intends to act on such beliefs. Therefore, the inclusion of a measure to assess the decision to trust, in addition to measures of trustworthiness, would provide a more comprehensive understanding of the trust process.

\section{Research aim and hypotheses}

This study aims to explore dimensions of trust (i.e. the precursors of trust in relation to trust itself) as they manifest in the dyadic relationship between employees in general - that is, without differentiating between specific demographic groups - and their leaders. The decision not to differentiate followers on the basis of more specific demographic sub-groups was based firstly on the need to compare trends with previous research in other countries which did not necessarily use such specific differentiations between sub-groups in their studies. Although it is acknowledged that members of the same race, gender, generation or any other demographic group may be inclined to use perceived similarities as a foundation for interpersonal trust, previous research established - quite contrary to popular belief - that the similarity-trust versus dissimilarity-distrust paradigm is inadequate to explain trust decisions (Lau, Lam \& Salamon, 2008; Williams, 2001). Lau et al. (2008) argue that trust decisions are not necessarily determined by perceived similarity of one specific demographic attribute only, but rather by an interaction effect of various categories with which one may identify (being white, female and belonging to a younger generation for instance). Welter and Alex (2012) furthermore highlight the fact that individuals who do not share a common cultural history or background can still trust each other through shared positive experiences of an alternative nature, such as through repeated successful business exchanges with each other over time. 
Although societal and organisational contingencies also influence trust decisions, this research does not focus on the macro- and meso level factors that influence trust but instead focuses only on an individual (micro) level of analysis. The study is confined to employee trust in direct leaders within private sector companies within a South African workplace context. It seeks to understand how specific antecedents of trust influence follower decisions to place trust in their leaders, explicitly in terms of their willingness to accept vulnerability and to take risks within the relationship.

This study seeks to make both a theoretical and an empirical contribution to the trust literature by examining antecedents of trust as based on the theoretical model developed by Mayer et al. (1995), in relation to the decision to trust, operationalised by various dimensions of vulnerability, as advanced by Gillespie $(2003,2012)$. The aim is to answer the following main research question: Which antecedent of trust - propensity or trustworthiness beliefs - can be regarded as the strongest predictor of the decision to trust, as evidenced by a willingness to rely on and disclose information to a trust referent? The research is primarily interested in establishing the above relationships within the context of dyadic interpersonal workplace relationships, specifically from the follower's point of view. The following hypotheses are proposed:

Hypothesis 1: Propensity is significantly and positively related to trustworthiness beliefs.

Hypothesis 2: Propensity is significantly and positively related to the reliance and disclosure dimensions of trust.

Hypothesis 3: Trustworthiness beliefs predict trusting behaviour.

Hypothesis 4: Trustworthiness mediates the relationship between propensity and trust.

Research concerning trust relationships on the interpersonal level, particularly when studied in dyadic relationships from the follower's point of view, is relatively scarce (Bachraim \& Hime, 2007). In addition, only a few researchers have attempted to link multiple dimensions of trust in the same study (Dirks \& Ferrin, 2002; Gillespie, 2003). In fact, the inclusion of multiple types of scales in a single study on trust is extremely rare (Colquitt et al., 2007). In the few cases in which more than one measuring instrument was included in the same study to link trustworthiness components to the decision to trust, the scales developed by Mayer and Davis (1999) were used, despite known problems with reliability experienced specifically with the four-item trust scale. Although Gillespie (2003) developed the Behavioural Trust Inventory (BTI) as an alternative trust measure, no studies, as far as the authors are aware, have ever attempted to use a combination of Mayer and Davis's well-known assessment of trustworthiness and Gillespie's measure of behavioural trust within the same study. This is even more surprising, considering the fact that the BTI was not only endorsed for future replication as an excellent measure for trust (McEvily \& Tortoriello, 2011) but was also specifically recommended for the exploration of the relationship between trustworthiness and the two dimensions of trust as differentiated by the BTI (Gillespie, 2003).

By including measures of main antecedents and the actual decision to trust in the same study, the multidimensionality of trust can be established more concretely. In addition, it would provide valuable information on trust-inducing interventions to promote decisions to trust. If the nature and strength of relationships between conditions for trust and trust itself could be determined, this knowledge could assist management in identifying risk patterns and devising more focused strategies to improve intra-organisational dyadic relationships within companies. Such knowledge can also help management decide on how and where resources should be employed to build workplace trust.

\section{Research design Research approach}

A cross-sectional survey design with questionnaires as the method of data collection was used to obtain information from the target population.

\section{Research method Participants}

Contextual factors for this study are limited to intraorganisational dyadic workplace relationships. The focus on followers and their direct leaders was selected due to its critical importance to performance outcomes. The study is further confined to workplace relationships within three Johannesburg Stock Exchange (JSE) listed private sector companies with an international footprint operating from the same geographical area of South Africa.

A convenience sample of 539 coded responses was drawn from an existing database, consisting of responses obtained from a survey amongst private sector companies in the petrochemical and raw materials industries. All respondents have a minimum qualification level of Grade 12 and are employed in positions at low, middle and senior levels of employment. The total sample $(n=540)$ consisted of 290 (54\%) men and 250 (46\%) women; 53\% were black and $47 \%$ were white. The highest age distribution is between 31 and 40 years of age (37.4\%), which is an indication that the employees are relatively in the middle of their working life. The majority of the sample group possesses a diploma or a bachelor's degree as their highest level of education (45\%) and the average length of service reporting to the current direct leader is more than 10 years.

\section{Measures}

The BTI (Gillespie, 2003) was used to measure trust between employees and their leaders. A key benefit of adopting this instrument is that it is a multidimensional measure of trust, which is particularly important since decisions regarding the willingness to render oneself vulnerable 
through trusting behaviour can manifest in different ways (McEvily \& Tortoriello, 2011). The BTI consists of two scales, namely reliance-based trust (items 1-5) and disclosure-based trust (items 6-10). Participants were requested to rate their willingness to demonstrate trusting behaviours towards their direct leader on a seven-point rating scale ranging from 1 (not at all) to 7 (completely). An example item measuring the first dimension, reliance-based trust, is 'How willing are you to depend on your leader to back you up in difficult situations?' (item 5). When establishing the construct validity of the BTI for the South African context, Heyns and Rothmann (in press) encountered problems with item 10 of the disclosure-based scale; therefore, this item was excluded from the second scale and only items 6-9 were used for the purpose of the current study. Heyns and Rothmann established configural, metric and scalar invariance for the specific items used in this research. An example item measuring the disclosure-based dimension is 'How willing are you to share your personal feelings with your leader?' (item 6). Previous research showed high reliability, ranging from 0.90 to 0.93 across samples and correlated between 0.61 and 0.71 across the samples. Validation was tested in cross-validation samples and supported the construct validity, divergent validity and predictive validity of the BTI (Gillespie, 2003).

Items of the Organisational Trust Instrument (OTI; Mayer \& Davis, 1999) were used to measure perceived trustworthiness of a direct leader (17 items) and propensity (six items, excluding the reversed items on this scale). Each item requires respondents to answer on a scale ranging from 1 (disagree strongly) to 5 (agree strongly). An example item measuring propensity is 'Most experts tell the truth about the limits of their knowledge'. Example items measuring ability, benevolence and integrity, respectively, are 'I feel very confident about management's skills' (item 4), 'My needs and desires are very important to management' (item 8) and 'Sound principles seem to guide management's behaviour'. During its initial standardisation, all subscales obtained acceptable reliability coefficients ranging from 0.93 for ability and 0.95 for benevolence to 0.96 for integrity (Mayer \& Davis, 1999).

\section{Data analysis}

The data analysis was carried out by means of Mplus version 7.3 (Muthén \& Muthén, 2014). The weighted least-squares with mean and variance adjustment (WLSMV) estimator was used to test the measurement and structural models. This estimator is robust; it does not assume normally distributed variables and it provides the best option for modelling categorical data (Brown, 2006). The following Mplus fit indices were used in this study: absolute fit indices, which included the chi-square statistic (the test of absolute fit of the model), the weighted root mean square residual (WRMR) and the root means square error of approximation (RMSEA), and incremental fit indices, which included the TuckerLewis Index (TLI) and the comparative fit index (CFI) (Hair, Black, Babin \& Andersen, 2010). The CFI also compares the hypothesised and independent models, but takes sample size into account. The TLI is a relative measure of covariation explained by the hypothesised model which has been specifically designed for the assessment of factor models. Critical values for good model fit have been recommended for the CFI and TLI to be acceptable above the 0.90 level (Wang \& Wang, 2012), although Hu and Bentler (1999) recommend a cut-off value of 0.95 . The RMSEA provides an indication of the overall amount of error in the hypothesised modeldata fit, relative to the number of estimated parameters (complexity) in the model. The recommended acceptable levels of the RMSEA should be 0.05 or less and should not exceed 0.08 . Hu and Bentler suggest a value of 0.06 to indicate acceptable fit. Chi-square difference tests were conducted to compare alternative nested structural models (Muthén \& Muthén, 2014).

Reliabilities ( $\rho$ ) of scales measured by items rated on a continuous scale were computed using a formula based on the sum of squares of standardised loadings and the sum of standardised variance of error terms (Raykov, 2009; Wang \& Wang, 2012). This was done as an alternative for Cronbach's alpha, which does not provide a dependable estimate of scale reliability when latent variable modelling is used.

To investigate the significance of hypotheses related to indirect effects, the procedure described by Hayes (2013) was used. Bootstrapping (with 10000 samples) was used to construct two-sided bias-corrected 95\% confidence intervals (CIs) so as to evaluate indirect effects.

\section{Research procedure}

The study was cleared by the local university's ethics committee. Next, the purpose and objectives of the study were stipulated on a cover letter and attached to a questionnaire, which was distributed in hard copy format to the participants. The cover letter stated that participation is completely voluntary and anonymous and that information obtained would be used for research purposes only. The name and contact details of the researcher were indicated on the cover letter to allow participants an opportunity to clarify queries and to request feedback on the results of the study.

\section{Results Testing measurement models}

The researchers tested a series of competing measurement models using confirmatory factor analyses (CFA) with Mplus to evaluate the distinctness of the measured variables. Four alternative models were considered.

The first model included three latent variables, namely (1) propensity (measured by six observed variables), (2) trustworthiness, consisting of three latent variables, namely ability (measured by six observed variables), benevolence (measured by five observed variables) and integrity (measured by six observed variables), and (3) trust consisting of two latent variables, namely reliance (measured by five observed variables) and disclosure (measured by four observed variables). The measurement of 
propensity and trust remained the same in model 2 (as in model 1). However, trust was measured by nine observed variables. The third model tested whether the three latent constructs of ability, benevolence and trustworthiness were superfluous in the measurement of trustworthiness. The measurement of propensity and trust remained exactly as in model 1, whilst trustworthiness was measured by 17 directly observed indicators which did not differentiate between ability, benevolence and integrity as separate factors of the trustworthiness construct. The fourth model consisted of 32 observed variables which measured one latent variable. Table 1 presents the results of these analyses.

The first model reflects that the respondents differentiate each factor from the others as the theory proposes. Although the chi-square value was statistically significant, model 1 provided a good fit to the data on three of the other four fit indices: $\chi^{2}(N=603)=1439.58, d f=456, p<0.001 ; \mathrm{CFI}=0.97$, $\mathrm{TLI}=0.96$, RMSEA $=0.05$ (95\% CI 0.056-0.063) and WRMR = 1.43. The results indicated that the relationship between each observed variable and its respective construct was statistically significant $(p<0.01)$, establishing the posited relationships amongst indicators and constructs (see Hair et al., 2010).

The reliability and correlation coefficients of the scales are reported in Table 2.

Table 2 shows that the reliability coefficients of all the scales were acceptable $(\rho \geq 0.70)$. Correlations between the trustworthiness subscales (i.e. ability, benevolence and integrity) ranged from 0.78 to 0.91 . Correlations of medium effect ( $r \geq 0.30$; Cohen, 1988) were found between the subscales of trustworthiness and propensity. Correlations of small effect were found between propensity and the trust subscales. The effect sizes of correlations between dimensions of trustworthiness and trust were large ( $r \geq 0.50$; Cohen, 1988).

\section{Testing structural models}

The structural model was tested by using latent variable modelling as implemented by Mplus, version 7.3 (Muthén \&
Muthén, 2014). Results indicated a good fit of the re-estimated model to the data: $\chi^{2}=1439.58, d f=456, p<0.001$; RMSEA $=$ 0.06 (90\% CI: $0.056,0.063), \mathrm{CFI}=0.97$, TLI $=0.96$ and WRMR $=$ 1.46. Two other competing models were also tested: model 2 (a partial mediation model) included paths from propensity to trustworthiness, and from trustworthiness to trust. Model 3 (a direct effects model) included paths from propensity to trust and from trustworthiness to trust. The following changes in chi-square $\left(\Delta \chi^{2}\right)$ were found: model 1 and model 2 $\Delta \chi^{2}=2.65, \Delta d f=1, p<0.1034$; models 1 and $3 \Delta \chi^{2}=53.39, \Delta d f=$ $1, p<0.0001$. Therefore model 1 was superior to model 2 and model 3. Figure 2 and Table 3 show the standardised path coefficients estimated by Mplus for the first structural model.

In model 3 (when the path of propensity to trustworthiness was fixed to zero), propensity had a direct effect on trust $(\beta=0.20, p=0.01)$. The explained variances in the observed variables measuring propensity ranged from 0.16 to 0.37 , indicating that this scale could be improved. The results provide partial support for hypothesis 1 , since a statistically significant effect of propensity on trustworthiness was observed $(\beta=0.34, p<0.01)$. Hypothesis 2 , which stated that positive correlations exist between propensity and the two sub-dimensions of trust, is also accepted, but although correlations were statistically significant, the effect sizes were small. As is evident from Table 3, propensity did not have a significant direct effect trust once trustworthiness was included in the model $(\beta=-0.07, p=0.10)$.

Table 3 furthermore shows that trust is predicted by the extent to which a trusting party perceives the trust target as trustworthy, as the path coefficient of trustworthiness was statistically significant and had the expected sign $(\beta=0.80$, $p<0.001)$. The explained variances related to this portion of the model were substantial (ability $R^{2}=0.67$; benevolence $R^{2}=0.87$, integrity $R^{2}=0.98$ ). Hypothesis 3 , stating that all three trustworthiness beliefs are significantly and positively related to trust, was therefore supported. Therefore, a trusting party's willingness to trust is predicted by the extent to which the trust target is perceived as being trustworthy.

TABLE 1: Fit statistics for the hypothesised competing measurement models.

\begin{tabular}{|c|c|c|c|c|c|c|}
\hline Model & Chi-square statistic & $d f$ & Tucker-Lewis index & Comparative fit index & Root mean square error of approximation & Weighted root mean square residual \\
\hline 1 & $1439.58 *$ & 456 & 0.96 & 0.97 & $0.06 *[0.056,0.063]$ & 1.43 \\
\hline 2 & 2087.26 & 458 & 0.94 & 0.94 & $0.08 *[0.073,0.080]$ & 1.82 \\
\hline 3 & 1969.38 & 458 & 0.94 & 0.95 & $0.07 *[0.071,0.077]$ & 1.73 \\
\hline 4 & 6416.85 & 464 & 0.78 & 0.80 & $0.15 *[0.140,0.149]$ & 3.38 \\
\hline
\end{tabular}

TABLE 2: Descriptive statistics, reliabilities and correlations of the scales

\begin{tabular}{lcccccc}
\hline Variable & Mean & Standard deviation & $\boldsymbol{\rho}$ & $\mathbf{1}$ & $\mathbf{2}$ & $\mathbf{3}$ \\
\hline 1. Propensity & 2.84 & 0.66 & 0.70 & - & - & - \\
2. Trustworthiness: Ability & 3.65 & 0.81 & 0.89 & 0.29 & - & - \\
3. Trustworthiness: Benevolence & 3.09 & 0.97 & 0.91 & 0.32 & 0.78 & - \\
4. Trustworthiness: Integrity & 3.22 & 0.73 & 0.79 & 0.34 & 0.83 & - \\
5. Trust: Reliance & 4.81 & 1.42 & 0.89 & 0.18 & 0.56 & - \\
6. Trust: Disclosure & 4.36 & 1.55 & 0.86 & 0.17 & - & - \\
\hline
\end{tabular}

All correlations are statistically significant: $p<0.01$. 


\section{Indirect effects}

To determine whether the relationship between propensity and trust was indirectly affected by perceived trustworthiness, the procedure explained by Hayes (2013) was used. Bootstrapping was used to construct two-sided bias-corrected $95 \%$ and $99 \%$ CIs so as to evaluate the significance of indirect effects. The lower and upper CIs are reported in Table 4.

Table 4 shows that the indirect effect of propensity on trust $(\beta=0.27)$ is significant. Also the $95 \%$ confidence intervals $(0.18,0.36)$ did not include zero. Hypothesis 4 is supported. Therefore trustworthiness mediates the relation between propensity and trust. Individuals with a higher propensity to trust will tend to have more positive perceptions regarding the trust target's trustworthiness in terms of ability, benevolence and integrity characteristics which, in turn, will promote the development of trust and the willingness to actively engage in trusting behaviours.

Taken together, the results suggest that the relationships posited in the model account for a substantial amount of the covariation in the data. The model accounts for $12 \%$ of the variance in trustworthiness and $61 \%$ of the variance in trust, lending more empirical support for the model's fit.

TABLE 3: Standardised regression coefficients of trustworthiness and propensity in predicting trust.

\begin{tabular}{lcccc}
\hline Variable & Estimate & Standard error & $\begin{array}{c}\text { Estimate to standard } \\
\text { error ratio }\end{array}$ & $p$ \\
\hline Trustworthiness & - & - & - & - \\
Propensity & 0.34 & 0.04 & 7.87 & $0.000^{* *}$ \\
Trust & - & - & - & - \\
Propensity & -0.07 & 0.04 & -1.65 & 0.103 \\
Trustworthiness & 0.80 & 0.03 & 28.90 & $0.000^{* *}$ \\
\hline$* *, p<0.01$ & & & &
\end{tabular}

\section{Discussion}

This study examined the dynamic interplay between trust propensity, trustworthiness beliefs and trust within the context of dyadic workplace relationships. In support of the trust model developed by Mayer et al. (1995), results confirmed the distinctness of propensity, trustworthiness and trust as separate main constructs. Trust was strongly influenced by trustworthiness beliefs. Assessments of the trust referent's integrity and benevolence appeared to be leading considerations. In contrast, propensity had a moderate indirect effect on trust (via trustworthiness).

Regarding the trustworthiness construct specifically, particularly high inter-correlations were noted between the benevolence and integrity subscales and the overall trustworthiness construct. Although this seemed to suggest a lack of differentiation, the standardised estimated model parameters for the three latent factors in the current model were all significant. Moreover, the fit indices for the preferred model were superior to those for a model in which the three factors were collapsed into one construct with 17 directly observed indicators of trustworthiness.

Integrity had the strongest effect on trust, followed by benevolence and ability, respectively. Propensity did not have an independent, direct impact on the decision to trust. This presents an interesting finding, since it implies that even if one has a consistent tendency to be willing to trust others across a broad spectrum of situations and targets, this disposition is only influential in impersonal situations and

TABLE 4: Indirect effect of propensity on trust.

\begin{tabular}{lcccc}
\hline Variable & Estimate & Standard error & \multicolumn{2}{c}{ 95\% confidence interval } \\
\cline { 3 - 5 } & & & Lower & Upper \\
\hline Trustworthiness & $0.27^{*}$ & 0.04 & 0.18 & 0.36 \\
\hline,$p<0.01$ & & & &
\end{tabular}

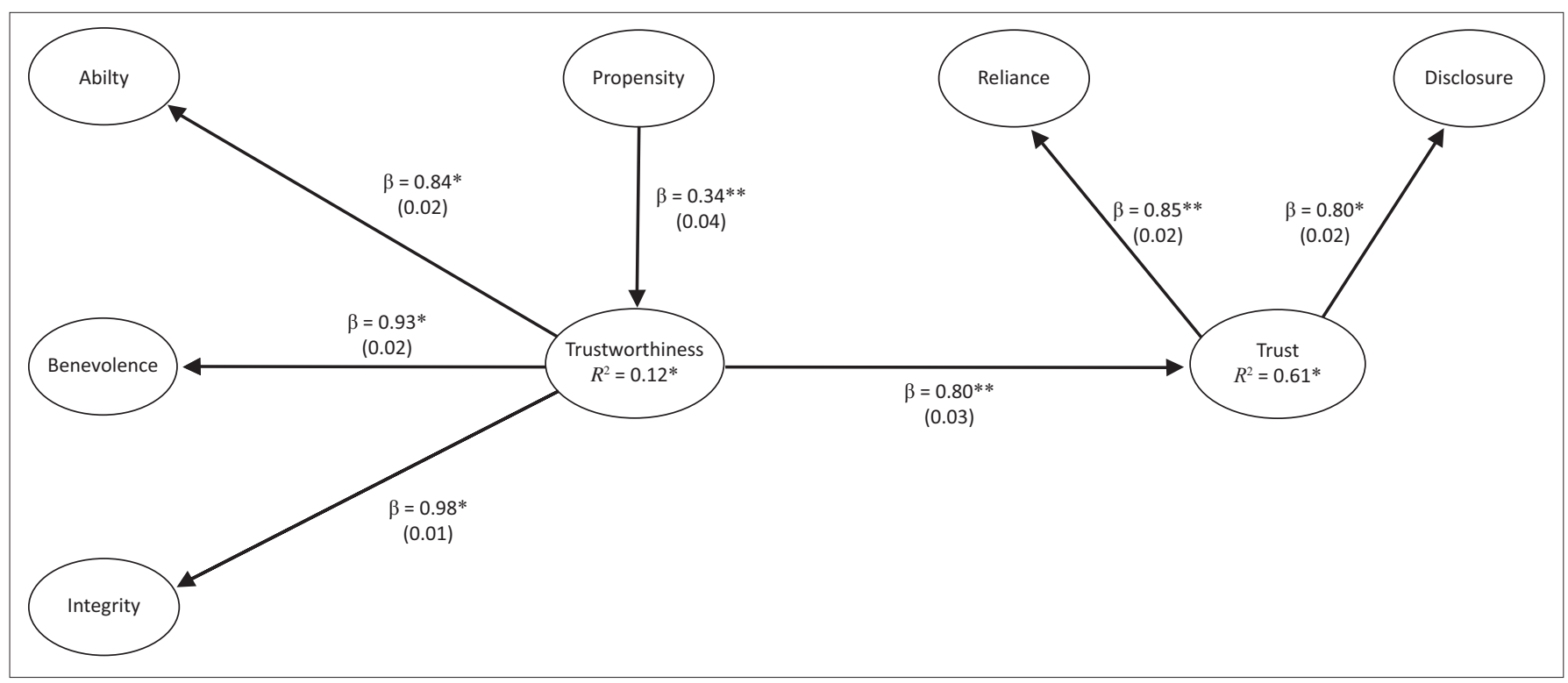

$*, p,<0.001$

FIGURE 2: The structural model (standardised solution with standard errors in parentheses). 
does not automatically imply willingness to trust within a specific relationship where the trust referent is known.

Analysis to determine indirect effects indicated that trustworthiness beliefs fully mediated the relationship between propensity and trust, thereby confirming that propensity is no longer persuasive in the decision to trust once trustworthiness could be established. It is, however, clear that propensity indirectly affects trust via trustworthiness beliefs: the observed relation between propensity and trustworthiness implies that individuals with a natural predisposition to trust others will be more inclined to perceive a specific trust referent as able, benevolent and having integrity. Therefore, higher levels of propensity will facilitate higher levels of perceived trustworthiness, which, in turn, will facilitate trust.

Findings regarding the influence of trustworthiness on trust are in line with Mayer et al.'s (1995) model and subsequent research (Gill et al., 2005; Mayer \& Gavin, 2005; Schoorman et al., 2007), confirming a strong positive relationship between trustworthiness beliefs and levels of trust. From a theoretical perspective, the observed correspondence between the benevolence and integrity constructs in our study is consistent with those found by previous researchers (Davis, Schoorman, Mayer \& Tan, 2000; Mayer \& Gavin, 2005; Schoorman et al., 2007) and provides further evidence of the intricate and idiosyncratic blend of the three trustworthiness factors, as initially outlined by Mayer et al. (1995). In congruence with the views of Dietz and Den Hartog (2006) regarding the conceptualisation of trustworthiness, the authors therefore believe that ability, benevolence and integrity may each be a significant consideration in themselves, but are also likely to act interdependently and in idiosyncratic combinations that will be substantially influenced by context.

In line with Mayer et al.'s (1995) model and subsequent research (Gill et al., 2005), the results of this study confirm that trustworthiness is a strong predictor of trust. Regarding the relative sequence of importance of the three trustworthiness components, previous research, such as that conducted by Davis et al. (2000) and Wasti and Tan (2010), for example, does not show equally significant relationships with trust in all instances. Davis et al. confirmed a relationship between all three components and trust, but regression analysis showed that these relationships were only significant in the case of benevolence and integrity; they attributed this finding to likely effects of multicollinearity. Wasti and Tan followed a qualitative research approach to study the relative importance of trust antecedents within collectivist countries and found that, in terms of overall antecedent categories, integrity was mentioned most frequently, followed by ability and benevolence.

It is, however, important to note that dissimilar measures for trust were used in our study as compared to previous research that mostly relied on Mayer and Davis's (1999) trust measure; therefore, comparisons between findings of the different studies need to be interpreted with caution. In fact, we are of the opinion that a meaningful comparison of this aspect to previous research requires that all contextual variables that might have influenced trust decisions within the various studies should be equitable. In support of this view, Sweeney (2010) for instance demonstrated that highvulnerability contexts caused followers to re-prioritise the basis for trust in their leader.

With respect to the relationship between trustworthiness beliefs and the decision to trust as conceptualised by a twofactor structure, Gillespie (2003) claims that the reliance dimension is more ability-based and therefore relies on the cognitive assessments of the trust referent, in contrast to the disclosure dimension which is more relationship-oriented and taps into more intuitive and emotionally driven motives to trust. She projected that beliefs about another's ability might be a stronger determinant of reliance-based trust, whereas assessments of benevolence would be a stronger determinant of disclosure-based trust. This study did not provide definitive support for these claims: whilst only a slightly stronger correspondence between ability beliefs and reliance-based trust was observed, the same could not be said of the relationship between perceived benevolence and disclosure-based trust.

Previous research regarding the effect of propensity on trust rendered mixed results (McKnight et al., 1998). This study indicated that propensity was not a strong predictor of trust, similar to findings of Mayer and Gavin (2005). In contrast, both Colquitt et al. (2007) and Searle et al. (2011) found that propensity remained important, even when trustworthiness was considered simultaneously. Interestingly, research by Gill et al. (2005) established that an individual's disposition to trust correlated with intention to trust when information about trustworthiness was ambiguous, but did not correlate with intention to trust when information about trustworthiness was clear. It is possible that future studies could shed more light on these apparent conflicting findings by controlling for more specific contextual variables such as the duration or stage of the relationship, or the perceived level of risk.

The observed relationship between propensity and trustworthiness resonates well with previous research suggesting that propensity could prejudice one's belief in the trustworthiness of others (Gill et al., 2005; Kosugi \& Yamagishi, 1998; McKnight et al., 1998). Similar to the findings of McKnight et al. (1998), this implies that individuals with high levels of generalised trust in others would not only selectively attend to information congruent with their trust disposition, but would also interpret new material according to their natural tendency (McKnight et al., 1998).

This study contributes to the promotion of workplace trust relationships as follows: results provide further empirical 
support for the theoretical trust model proposed by Mayer et al. (1995) as applied locally (i.e. outside the traditional First World countries), specifically with regard to the distinctness of propensity, trustworthiness and trust as separate main constructs of the model. The fact that propensity and trustworthiness beliefs of followers within the current sample group show relatively similar patterns to those previously established in other countries seems to support the idea that followers, at least as far as their considerations for trusting leaders are concerned, may not be so different as one might be inclined to presume after all.

In this study, the dimensionality of trust was investigated by combining a measure of trustworthiness (Mayer \& Davis, 1999) with the measure of behavioural trust as developed by Gillespie (2003) within the same study. Since the latter serves as a closer proxy of actual trust than measures of trustworthiness (Gillespie, 2012; McEvily \& Tortoriello, 2011), such a design enabled the researchers to obtain a more precise assessment of how trustworthiness beliefs might influence the decision to trust. Since results showed that all three trustworthiness beliefs predicted both reliance-based and disclosure-based trust decisions directly, leaders should focus their effort and resources on advancing their integrity, benevolence and abilities as perceived by others. Findings revealed that higher levels of propensity will facilitate higher levels of perceived trustworthiness; therefore, it seems worthwhile to explore ways and means in which generalised trust in others can be cultivated.

Because individual's perceptions were determined, selfreport scales were used. We tried to curtail the effect of same-source bias (Podsakoff, MacKenzie, Lee \& Podsakoff, 2003) that is a disadvantage of this method by clearly separating all the main constructs in the questionnaire and to add individualised instructions to each section, but we acknowledge that common method variance could still have influenced findings. Finally, the use of a cross-sectional survey design limits the generalisability of findings beyond the sample group.

\section{Recommendations}

Leaders should realise that their attitudes and behaviour have a decisive impact on trust formation processes: only if they are being perceived as trustworthy, will followers be likely to respond by engaging in trusting behaviours towards them. Tools to assess followers' perceptions of the trustworthiness of the leader may therefore provide useful feedback that can guide leaders in terms of their personal development plans.

Trustworthiness beliefs can be recognised as follows: integrity, as the most dominant predictor of trust in this study, is often conceptually associated with fairness, consistency and promise fulfilment (Colquitt et al., 2007) as well as with accountability, justice and value congruence (Burke et al., 2007). Previous research (Skarlicki \& Latham, 2005) has established that supervisors can be trained to adhere to justice principles. Leaders therefore need not strive to convey these behaviours merely intuitively; they can attend training courses to sensitise them to justice principles.

Behavioural indicators suggest that integrity is essentially judged by the extent to which the trust target adheres to sound moral and ethical principles (Colquitt et al., 2007), especially when these are perceived to be similar to those of the trustor (Gilstrap \& Collins, 2012). According to Gillespie and Mann (2004), sharing of common values promotes trusting behaviour because the leader is unlikely to act contrary to these values. This increases predictability and perceived risk and uncertainty are lowered as a result. It is therefore advised that leaders should take time to explain to followers what the values and beliefs are that guide their actions and to promote tolerance for different perspectives on this topic. In addition, they should ensure that the tangible policies, workplace procedures and recognition systems that are put in place are transparent and consistent with their views.

Benevolence beliefs can be promoted by showing genuine concern and commitment to the development of followers, by efforts to increase followers' self-esteem and by initiatives to nurture and leverage talent (Burke et al., 2007; Caldwell \& Dixon, 2009). Such goodwill would also be exemplified by creating and sustaining supportive contexts, a consultative decision-making style, coaching and mentoring, and certain transformational and transactional behaviours (Burke et al., 2007). Leaders can also attend specific leadership training and development programmes to equip them with skills in consulting and involving members in important decisions (Gillespie \& Mann, 2004).

Leaders who want to be perceived as competent should advance their technical and management skills (Mayer \& Davis, 1999). This implies that they should augment both the knowledge and skills to do a specific job (cognitive and physical capacities), as well as the interpersonal skills and general wisdom (emotional intelligence) needed to succeed in a workplace environment (Colquitt et al., 2007, 2011). Ability could also be promoted by setting compelling direction and creating enabling structures to promote effective task execution, disseminating relevant information and setting functional norms to help employees adapt to particular situations (Burke et al., 2007) and by demonstrating that one is capable of delivering results (Tseng, Chen \& Chen, 2005).

Future studies exploring the relationship between propensity, trustworthiness and trust could improve the current knowledge base by controlling for more macro-level and micro-level contextual effects that may affect the trust decision. Schoorman et al. (2007), for instance, believe that propensity is heavily influenced by personality, experiences and culture. Schoorman et al. suggest that cultural orientations could influence the relative importance assigned to ability, benevolence and integrity as conditions for trust: more 
action-oriented cultures possibly place more emphasis on ability as condition to trust, whilst more relationshiporientated cultures may be more prone to be guided by indicators of a trustee's benevolence. Costigan et al. (2007) furthermore indicate that collectivist cultures put a higher premium on relationship-based conditions for trust within dyadic workplace relationships than respondents from individualistic cultures.

Although the differentiation of propensity and trustworthiness perceptions in relation to trust decisions as perceived by specific demographic sub-groups such as race, gender and generational groupings fell beyond the scope of the current study for reasons explained earlier, it is acknowledged that several contextual factors not considered in this study may indeed also influence the trust formation process. In view of the fact that both individualistic and collectivistic cultures contribute to the diversity of the South African workplace for example, it makes sense that cultural background should be included as one particular variable that should be controlled for in future studies on this topic. Future research should specifically determine whether individuals from culturally diverse backgrounds differ in their respective levels of propensity and also whether they differ in their relative preferences assigned to specific antecedents considered when making the decision to trust. It would however be necessary to control for the interaction effect of several variables such as a specific relationship's history, stage of development and cues in the immediate setting (Rousseau et al., 1998) within the same study as well.

By controlling for more contextual variables within studies, researchers can determine commonalities between contexts so that more meaningful comparisons across studies become possible. Since the disclosure-based dimension of trust is associated with an emotional, relationship-based form of trust (Gillespie, 2003), and as such is regarded as a more robust and resilient form of trust (McShane \& Von Glinow, 2010), future studies should uncover which trustworthiness beliefs would best promote this dimension of trust. A qualitative research approach is probably the best way to do this; by doing so, researchers can help both leaders and their followers to fill the trust gap by a continued search for new pathways to build and repair trust between employees and their leaders.

\section{Acknowledgements Competing interests}

The authors declare that they have no financial or personal relationships that may have inappropriately influenced them in writing this article.

\section{Authors' contributions}

M.H. (North-West University) was the project leader and gathered the data. S.R. (North-West University) was the promoter of the study and conducted the data analysis. Both authors contributed to writing the manuscript.

\section{References}

Bachraim, J., \& Hime, P. (2007). The dimensionality of workplace interpersonal trust and its relationship to workplace affective commitment. SA Journal of Industrial Psychology, 33, 43-48. http://dx.doi.org/10.4102/sajip.v33i3.394

Ball, J. (2009). The currency of trust: What business leaders can learn from the extreme poor. Ivey Business Journal, 73.

Bhattacharya, R., Devinney, T.M., \& Pillutla, M.M. (1998). A formal model of trust based on outcomes. The Academy of Management Review, 23, 459-472. http://dx.doi.org/10.2307/259289

Brown, T. (2006). Confirmatory factor analysis for applied research. New York: Guildford. http://dx.doi.org/10.1080/00036810600603377

Burke, C.S., Simms, D.E., Lazzara, E.H., \& Salas, E. (2007). Trust in leadership: A multi-level review and integration. The Leadership Quarterly, 18, 606-632. http://dx.doi.org/10.1016/j.leaqua.2007.09.006

Burke, R.J. (2006). Why leaders fail: Exploring the dark side. International Journal of Manpower, 27, 91-100. http://dx.doi.org/10.1108/01437720610652862

Caldwell, C., \& Dixon, R.D. (2009). Love, forgiveness and trust: Critical values of the modern leader. Journal of Business Ethics, 93, 91-101. http://dx.doi.org/10.1007/ modern leader. Journal

Cohen, J. (1988). Statistical power analysis for the behavioral sciences. (2nd edn.). Hillsdale, NJ: Lawrence Erlbaum Associates. http://dx.doi.org/10.1002/ bs.3830330104

Colquitt, J.A., Lepine, J.A., \& Wesson, M.J. (2009). Organizational behavior: Improving performance and commitment in the workplace. New York: McGraw-Hill/Irwin.

Colquitt, J.A., Lepine, J.A., \& Wesson, M.J. (2011). Organizational behavior: Improving performance and commitment in the workplace (2nd edn.). New York: McGrawHill/Irwin

Colquitt, J.A., \& Rodell, J.B. (2011). Justice, trust, and trustworthiness: A longitudinal analysis integrating three theoretical perspectives. Academy of Management Journal, 54, 1183-1206. http://dx.doi.org/10.5465/amj.2007.0572

Colquitt, J.A., Scott, B.A., \& LePine, J.A. (2007). Trust, trustworthiness, and trust propensity: A meta-analytic test of their unique relationships with risk taking and job performance. Journal of Applied Psychology, 92, 909-927. http://dx.doi. org/10.1037/0021-9010.92.4.909

Costigan, R.D., Insinga, R.C., Berman, J.J, Ilter, S.S., Kranas, G., \& Kureshov, V.A. (2007). A cross-cultural study of supervisory trust. International Journal of Manpower, 27, 764-787. http://dx.doi.org/10.1108/01437720610713549

Covey, S.M.R. (2006). The speed of trust: The one thing that changes everything. New York: Simon \& Schuster.

Davis, J.H., Schoorman, F.D., Mayer, R.C., \& Tan, H.H. (2000). The trusted general manager and business unit performance: Empirical evidence of a competitive advantage. Strategic Management Journal, 21, 563-576. http://dx.doi. org/10.1002/(SICI)1097-0266(200005)21:5\%3C563::AID-SMJ99\%3E3.0.CO;2-0

Dietz, G., \& Den Hartog, D.N. (2006). Measuring trust inside organizations. Personnel Review, 35, 557-588. http://dx.doi.org/10.1108/00483480610682299

Dirks, K.T., \& Ferrin, D.L. (2002). Trust in leadership: Meta-analytic findings and implications for research and practice. Journal of Applied Psychology, 8, 611-628. http://dx.doi.org/10.1037/0021-9010.87.4.611

Douglas, C., \& Zivnuska, S. (2008). Developing trust in leaders: An antecedent of firm performance. SAM Advanced Management Journal, 73, 20-27.

Esterhuizen, W., \& Martins, N. (2008). Organisational justice and employee responses to employee equity. SA Journal of Labour Relations, 32(2), 66-85.

Gambetta, D. (2008). Can we trust trust? In Bachman, R., \& Zaheer, A. (Eds.), Landmark papers on trust, (Vol. 1, pp. 133-157). Cheltenham, UK: Edward Elgar.

Gill, H., Boies, K., Finegan, J.E., \& McNally, J. (2005). Antecedents of trust: Establishing boundary conditions for the relation between propensity to trust and intention to trust. Journal of Business and Psychology, 19, 287-302.

Gillespie, N. (2003). Measuring trust in work relationships: The Behavioral Trust Inventory. In Proceedings of the Academy of Management Conference. Seattle, WA.

Gillespie, N. (2012). Measuring trust in organizational context: An overview of surveybased measures. In F. Lyon, G. Möllering, \& M.N.K. Saunders (Eds.), Handbook of research methods on trust (pp. 175-188). Cheltenham, UK: Edward Elgar Publishing.

Gillespie, N., \& Mann, L. (2004). Transformational leadership and shared values: The building blocks of trust. Journal of Managerial Psychology, 19, 588-607. http:// dx.doi.org/10.1108/02683940410551507

Gilstrap, J.B., \& Collins, B.J. (2012). The importance of being trustworthy: Trust as a mediator of the relationship between behaviors and employee job satisfaction Journal of Leadership and Organizational Studies, 19, 152-163. http://dx.doi. org/10.1177/1548051811431827

Google Scholar. (2013). Search results. Retrieved July 18, 2013, from http://scholar google.com/scholar?q=mayer+trust\&hl=en\&lr=\&btnG=Search

Green, C. (2012). Why trust is the new core of leadership. Retrieved April 16, 2015

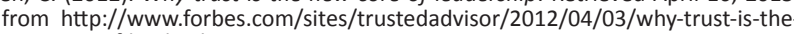
new-core-of-leadership

Grey, C., \& Garstin, C. (2001). Trust, control and post bureaucracy. Organization Studies, 22, 229-250. http://dx.doi.org/10.1177/0170840601222003

Greyvenstein, H., \& Cilliers. F. (2012). Followership experiences of organisational leadership: A systems psychodynamic perspective.SA Journal of Industrial Psychology/SA Tydskrif vir Bedryfsielkunde, 38(2), Art. \#1001, 10 pages. http:// dx.doi.org/10.4102/sajip.v38i2.1001 
Hair, J.F., Black, W.C., Babin, B.J., \& Andersen, R.E. (2010). Multivariate data analysis: A global perspective. Upper Saddle River, NJ: Pearson. http://dx.doi.org/10.1016/j. jmva.2009.12.014

Hayes, A.F. (2013). Introduction to mediation, moderation, and conditional process analysis. London: The Guilford Press.

Heyns, M., \& Rothmann, S. (in press). Comparing trust levels of male and female managers: Measurement invariance of the Behavioural Trust Inventory. South African Journal of Psychology.

Hu, L.T., \& Bentler, P.M. (1999). Cut-off criteria for fit indexes in covariance structure analysis: Conventional criteria versus new alternatives. Structural Equation Modeling: A Multidisciplinary Journal, 6, 1-55. http://dx.doi. org/10.1080/10705519909540118

Kenexa. (2012). High Performance Institute WorkTrends report. Retrieved April 16, 2015, from http://www.kenexa.com/ThoughtLeadership/WorkTrendsReports/TrustMatters

Kosugi, M., \& Yamagishi, T. (1998). General trust and judgments of trustworthiness. Japanese Journal of Psychology, 69, 349-357. http://dx.doi.org/10.4992/ jjpsy.69.349

Kramer, R.M. (2006). Organizational trust: A reader. New York: Oxford University Press.

Lau, D.C., Lam, L.W., \& Salamon, S.D. (2008). The impact of relational demographics on perceived managerial trustworthiness: Similarity or norms? The Journal of Socia Psychology, 148, 187-208. http://dx.doi.org/10.3200/SOCP.148.2.187-209

Mayer, R.C., \& Davis, J.H. (1999). The effect of the performance appraisal system on trust for management: A field Quasi-experiment. Journal of Applied Psychology 84, 123-136. http://dx.doi.org/10.1037/0021-9010.84.1.123

Mayer, R.C., Davis, J.H., \& Schoorman, F.D. (1995). An integrative model of organizational trust. Academy of Management Review, 20, 709-734. http://
dx.doi.org/10.2307/258792

Mayer, R.C., \& Gavin, M.B. (2005). Trust in management and performance: Who minds the shop while the employees watch the boss? Academy of Management Journal, 48, 874-888. http://dx.doi.org/10.5465/AMJ.2005.18803928

McEvily, B., Perrone, V., \& Zaheer, A. (2003). Trust as an organizing principle. Organization Science, 14, 91-103. http://dx.doi.org/10.1287/orsc.14.1.91.12814

McEvily, B., \& Tortoriello, M. (2011). Measuring trust in organisational research Review and recommendations. Journal of Trust Research, 1, 23-63. http://dx.doi. org/10.1080/21515581.2011.552424

McKnight, D.H., Cumming, L.L. \& Chervany, N.L. (1998). Initial trust formation in new organizational relationships. The Academy of Management Review, 23, 473-490. http://dx.doi.org/10.2307/259290

McShane, S.L., \& Von Glinow, M.A. (2010). Organizational behaviour: Emerging knowledge and practice for the real world. New York: McGraw-Hill/Irwin.

Möllering, G. (2006). Trust: Reason, routine, reflexivity. Oxford, UK: Elsevier Science.

Muthén, L.K., \& Muthén, B.O. (2014). Mplus user's guide. (7th edn.). Los Angeles, CA: Muthén \& Muthén.

Nooteboom, B., Berger, H., \& Noorderhaven, N.G. (1997). Effects of trust and governance on relational risk. Academy of Management Journal, 40, 308-338. http://dx.doi.org/10.2307/256885

Pienaar, C. (2009). The role of self-deception in leadership ineffectiveness: A theoretical overview. South African Journal of Psychology, 39, 133-141. http:// dx.doi.org/10.1177/008124630903900112

Podsakoff, P.M., Mackenzie, S.B., Lee, J., \& Podsakoff, N.P. (2003). Common method bias in behavioral research: A critical review of the literature and recommended remedies. Journal of Applied Psychology, 88, 879-903. http://dx.doi. org/10.1037/0021-9010.88.5.879

Raykov, T. (2009). Evaluation of scale reliability for unidimensional measures using latent variable modeling. Measurement and Evaluation in Counseling and Development, 42, 223-232. http://dx.doi.org/10.1177/0748175609344096
Rousseau, D.M., Sitkin, S.B., Burt, R.S., \& Camerer, C. (1998). Not so different after all: A cross-discipline view of trust. The Academy of Management Review, 20, 393-404. http://dx.doi.org/10.5465/AMR.1998.926617

Salamon, S.D., \& Robinson, S.L. (2008). Trust that binds: The impact of collective felt trust on organizational performance. Journal of Applied Psychology, 93, 593-601. http://dx.doi.org/10.1037/0021-9010.93.3.593

Schoorman, F.D., Mayer, R.C., \& Davis, J.H. (2007). An integrated model of organizational trust: Past, present, and future. The Academy of Management Review, 32, 334-354. http://dx.doi.org/10.5465/AMR.2007.24348410

Searle, R., Den Hartog, D.N., Weibel, A., Gillespie, N., Six, F., Hatzakis, T. et al. (2011). Trust in the employer: The role of high-involvement work practices and procedural justice in European organizations. The International Journal of Human Resource Management, 22, 1069-1092. http://dx.doi.org/10.1080/09585192.20 11.556782

Skarlicki, D.P., \& Latham, G.P. (2005). How can training be used to foster organizational justice? In J. Greenberg, \& J.A. Colquitt (Eds.), Handbook of organizational justice (pp. 499-522). Mahwah, NJ: Erlbaum.

Steinman, N., \& Martins, N. (2009). South African teams: Study reveals ten reasons for failure. People's Dynamic Development. Retrieved from http://www. peoplesdynamic.co.za/understanding_south_african_teams.htm

Sweeney, P.J. (2010). Do soldiers re-evaluate trust in their leaders prior to combat operations? Military Psychology, 22, 70-88. http://dx.doi.org/10.1080/ 08995601003644312

Sydow, J. (2008). Understanding the constitution of organizational trust. In R. Bachman, \& A. Zaheer (Eds.), Landmark papers on trust, (Vol. 1, pp. 362-383). Cheltenham, UK: Edward Elgar Publishing Ltd.

Tan, H., \& Lim, A. (2009). Trust in co-workers and trust in organizations. The Journal of Psychology, 143(1), 45-66. http://dx.doi.org/10.3200/JRLP.143.1.45-66

Tichy, N.M., \& Bennis, W.G. (2007). Judgement: How winning leaders make great calls. New York: The Penguin Group.

Tseng, H., Chen, T., \& Chen, F. (2005). Constructing and testing a model of trustworthiness, trust behaviour and organizational identification. The Journal of Nursing Research, 13, 293-330. http://dx.doi.org/10.1097/01. JNR.0000387552.88575.a7

Von der Ohe, H., \& Martins, N. (2010). Exploring trust relationships during times of change. SA Journal of Human Resource Management, 8, 1-9. http://dx.doi. org/10.4102/sajhrm.v8i1.256

Wang, J., \& Wang, X. (2012). Structural equation modeling: Applications using Mplus. Chichester, UK: Wiley. http://dx.doi.org/10.1002/9781118356258

Wasti, S.A., \& Tan, H.H. (2010). Antecedents of supervisor trust in collectivist cultures: Evidence from Turkey and China. In M.N.K. Saunders, D. Skinner, G. Doetz, N. Gillespie, \& R.I. Lewicki (Eds.), Organizational trust: A cultural perspective (pp. 331-335). Cambridge, UK: Cambridge University Press. http://dx.doi. org/10.1017/СВO9780511763106.013

Wasti, S.A., Tan, H.H., Brower, H.H., \& Önder, Ç. (2007). Cross-cultural measurement of supervisor trustworthiness: An assessment of measurement invariance across three cultures. The Leadership Quarterly, 18, 477-489. http://dx.doi. org/10.1016/j.leaqua.2007.07.004

Welter, R., \& Alex, N. (2012) Researching trust in different cultures. In F. Lyon, G. Möllering, \& M.N.K. Saunders (Eds.), Handbook of research methods on trust (pp. 50-59). Surrey, UK: Edward Elgar Publishing.

Werbel, J.D., \& Henriques, P.L. (2009). Different views of trust and relational leadership: Supervisor and subordinate perspectives. Journal of Managerial Psychology, 24 780-796. http://dx.doi.org/10.1108/02683940910996798

Williams, M. (2001). In whom we trust: Group membership as an affective context for trust development. Academy of Management Review, 26, 377-396. http://dx.doi. org/10.2307/259183 